\title{
POINT CLOUD SEGMENTATION OF GULLY BASED ON CHARACTERISTIC DIFFERENCE USING AIRBORNE LIDAR DATA
}

\author{
Ying $\mathrm{Xu}^{\mathrm{a}, \mathrm{b}, *}$, Dongjie Yue ${ }^{\mathrm{b}}$, Peipei $\mathrm{He}^{\mathrm{c}}$ \\ ${ }^{\text {a }}$ Institute of Resources and Environment, Henan University of Economics and Law, Zhengzhou, China - xuying@ncwu.edu.cn \\ ${ }^{b}$ School of Earth Sciences and Engineering, Hohai University, NanJing,China- yuedongjie@hhu.edu.cn \\ ${ }^{c}$ Institute of Resources and Environment, North China University of Water Resources and Electric Power, Zhengzhou, China - \\ he_pei@whu.edu.cn
}

KEY WORDS: Gully, airborne LIDAR, point cloud, curvature, normal vector, segmentation

\begin{abstract}
:
The point cloud segmentation of gullies with high accuracy lays the groundwork for the gully parameter extraction and developing models. A point cloud segmentation method of gullies based on characteristic difference from airborne LIDAR is proposed. Firstly, point cloud characteristics of gullies are discussed, and then differences in surface features are obtained based on different scales after preprocessing of the point cloud. Initial gullies are segmented from point clouds combined with a curvature threshold. Finally, real gully point clouds are obtained based on the clustering analysis. The experimental results demonstrate that gullies can be detected accurately with airborne LIDAR point clouds, and this method provides a new idea for quantitative evaluation of gullies.
\end{abstract}

\section{INTRODUCTION}

As a common type of soil erosion, gullies have an important position in soil erosion, and can lead to big volume of soil erosion (Pimentel, 1995) and (Wu, 2005). With the development of earth observation technologies and point cloud data-processing ability, airborne light detection and ranging (LIDAR) technology provides new technical ways to obtain earth spatial information with high space-time resolution (Meng, 2010). However, the huge number of point clouds, feature differences, and the complexity of natural terrains severely restrict the development of the data post-processing technology of airborne LIDAR. It is difficult to find the apparent surface characteristics of gullies as they are embedded in the earth's surface, unlike buildings, trees, roads, and bridges, coastlines (Zhu, 2014), which have obvious surface features. At the same time, the variation range, the gully-forming process, and the irregularity of appearance add to the difficulty of gully detection (Filin, 2014).

When LIDAR technology first appeared, Betts constructed a digital elevation model (DEM) of LIDAR data to extract local morphological information of valleys, the parameters of river bank erosion were determined, and a water loss and soil erosion model was established (Betts, 1999). Perroy combines airborne laser radar with ground laser radar to assess gully erosion, and airborne technology is more suitable for a wide range of gully monitoring, because of the limitations of scanning erosion grooves and sides by ground laser radar (Perroy, 2010). Evans proposes the extraction of the gully from a high-precision DEM to determine the gully depth based on the edge of the gully, and the whole geomorphological map of the gully is obtained (Evans, 2010). Mason obtains high gradient values for the edge detection operator in view of the point cloud; the method is suitable for a smooth surface area, but there are some limitations in complicated terrain (Mason, 2006). Rutzinger proposes an object driven approach for detection of points of interest based on the maximum curvature segmentation algorithm; this approach is suitable for deep and wide contour features, but the effect is not good for gully regions with textural complexity (M RA, 2014).

The above gully detection methods, which have a few limitations in the extraction of gullies with micro-morphological characteristics, are mostly based on
DEMs. Thus, this paper puts forward a method to extract gully information based on differences in surface characteristics at different scales. Considering gullies located in mountainous areas with complex surface characteristics, this paper segments the gully point cloud based on the normal vector and the changes of curvature, which improves the existing edge segmentation algorithm based on the point cloud.

\section{ANALYSIS OF CHARACTERISTICS OF POINT CLOUD}

The basic morphology of a gully is the profile and slope, and the section is generally a $\mathrm{U}$ or $\mathrm{V}$ shape, which can reflect the three-dimensional (3D) form of gully through both sides of the ditch along the route and the $U$ groove on both sides (or the bottom line of a V-shaped groove) (Zhang, 2015). Under hydraulic and gravitational action, a gully often has random characteristics because of the trench wall fighting force and deep valley (Baruch, 2011).

In short, the point cloud characteristics of gullies mainly comprise the following aspects. Firstly, in terms of the shape of an erosion gully, the ditch along the point cloud shows irregular, asymmetrical characteristics and an uneven distribution of trench wall point cloud density, the extraction of the gully becomes more difficult, and the difference in point cloud height between the boundary and the bottom of the trench is obvious. Secondly, due to the points of difference from other features such as buildings, trees, roads, bridges which have obvious surfaces features, it is obviously difficult to form the surface characteristics of the gully as they are embedded in the earth's surface, and erosion usually occurs in mountainous areas with sloping terrain and very irregular terrain characteristics. Moreover, the difficulty of detection is increased by the dynamic changes of the gully.

\section{CHARACTERISTIC DIFFERENCE SEGMENTATION METHOD}

The characteristic difference segmentation method means that the gully point cloud is obtained by choosing different radii, obtaining different surface characteristic parameters, and setting a certain segmentation threshold based on the gully in the changes of earth's surface (Douillard, 2010). The surface 
geometry information refers to the amount of data that can reflect the surface local features and mainly includes the surface normal vector, Gaussian curvature, mean curvature, surface changes, and so on, which reflect the trend of the surface normal vector, while the Gaussian curvature, mean curvature, and surface change reflect the degree of curvature of the surface (Lam, 2012).

\subsection{Normal vector and curvature estimation}

The normal vector reflects the geometric characteristics of the curved surface at some point, and the normal vectors of the point cloud are mainly determined through the neighborhood. The original point clouds do not have the normal vector (Tang, 2002). Therefore, the normal vectors are approximately equal to the normal vectors estimation on the tangential plane which is obtained by the neighborhood of the points, for each point in the point cloud $p_{i}=\left(x_{i}, y_{i}, z_{i}\right)^{T}$, through $\mathrm{k}$ adjacent points to the nearest, and each point $p_{i}$ of the covariance matrix can be expressed as:

$\mathrm{C}=\frac{1}{k}\left(p_{i}-\bar{p}\right) \cdot\left(p_{i}-\bar{p}\right)^{T}, C \cdot \vec{v}_{j}=\lambda_{j} \cdot \vec{v}_{j}, j \in\{0,1,2\}$

$\mathrm{k}$ is the total number of points in the field of the point $p_{i}, \bar{p}$ is the field of all points of the centre of mass, $\lambda_{j}$ is the jth characteristic value of the covariance matrix, and $\vec{v}_{j}$ is the jth eigenvector of the covariance matrix. When the minimum eigenvalue $\lambda_{1}$ is zero, the corresponding eigenvector $v_{1}$ is the normal vector of the point $p_{i}$.

The problem of the sign of the normal vector cannot be solved by the mathematical method, which means that the direction of the normal vector is arbitrary. To solve this problem, we need to adjust the consistency of the normal vector and select a view point $v_{p}$ that satisfies the equation:

$$
\overrightarrow{n_{l}} \cdot\left(v_{p}-p_{i}\right)>0
$$

The point cloud surface curvature calculated by formula (3) is:

$$
\sigma=\frac{\lambda_{0}}{\lambda_{0}+\lambda_{1}+\lambda_{2}}
$$

\subsection{Characteristics difference principle}

Characteristic difference treatment provides a multi-scale and highly efficient method for the processing of a large amount of disordered 3D point cloud data. This method uses each point of the set of $\mathrm{P}$ as the center, and choosing two different radii $r_{1}$ and $r_{2} \quad r_{1}>r_{2}$, we will get two different normal vectors $n_{1}\left(p_{i}, r_{1}\right), n_{2}\left(p_{i}, r_{2}\right)$ and curvature values. The selection of radius size depends on the point density. When point density is higher, the radius is smaller, point density is lower, the radius is larger. In general, the neighborhood point number determined by the radius floating up and down between 20 is better. The characteristic difference calculated by formula (4) and the sketch map is shown in Figure 1.

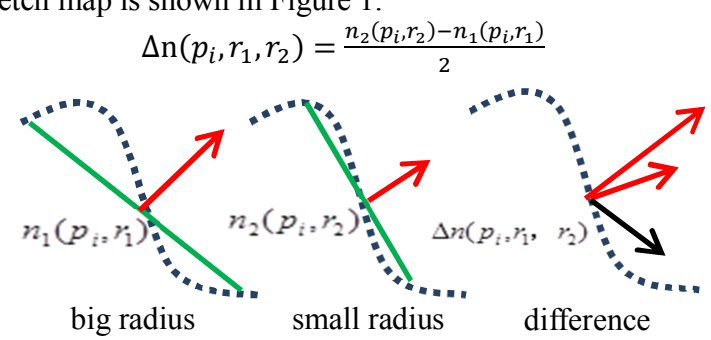

Figure 1. Characteristic difference

\subsection{Gully extraction based on characteristic difference}

The main work of gully extraction based on the characteristic difference is on the estimation of characteristic difference. In this paper, neighbouring points are determined according to a fixed distance. The distance can be selected according to the resolution of the point cloud (the size of the point spacing) to allow flexibility. As a general rule, a number of field points between 10 and 20 is relatively good. The extraction of the gully based on the characteristic difference is specified as follows:

1) Neighbourhood points are obtained by selecting radius $r_{1}$. The normal vector and the curvature values of the point cloud are obtained by the principal component analysis method, and the normal vector is adjusted consistently.

2) Neighbourhood points are obtained by selecting radius $r_{2}$. The normal vector and curvature values of the point cloud are obtained by the principal component analysis method, and the normal vector is adjusted consistently $r_{1}>r_{2}$.

3) The characteristic difference of each point value is calculated depending on the normal vector and curvature.

4) Based on the point cloud characteristics of the gully, the error threshold is set as (0.25), and difference values bigger than the threshold are extracted.

5) Based on the spatial clustering method according to dual distance $(3 \mathrm{~m})$, some of points having a characteristic difference are eliminated to obtain the point cloud of the gully.

Therefore, the flow chart of the gully segmentation algorithm based on surface characteristic differences is shown in Figure 2.

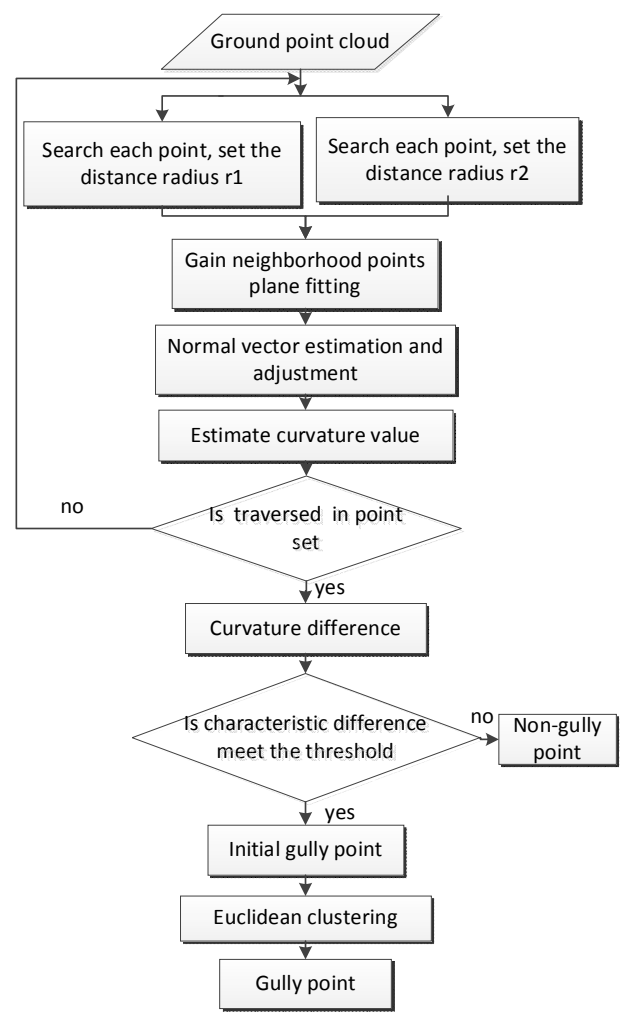

Figure 2. The flow chart of gully segmentation algorithm

\section{EXPERIMENT AND ANALYSIS OF RESULTS}

\subsection{The data}

In order to test the feasibility of the algorithm, it is implemented in the environment of $\mathrm{VC}++$. The experimental data are taken from certain areas of Jiayuguan city, Gansu province, and are obtained by the Gigel 780 system. The original point clouds include 1959273 points; this number 
decreases to 1940357 points, called ground points, after noise processing and filtering. The average density of the point cloud is 8 points $/ \mathrm{m}^{2}$, and the $3 \mathrm{D}$ diagram is shown in Fig. 3. From the diagram, it can be seen that there are lots of noise points in the original experimental data and the surface features mainly include power lines, buildings, sparse vegetation, and so on. Then the power lines, buildings, and sparse vegetation are mostly removed after noise processing and filtering, and the terrain features are kept perfectly.

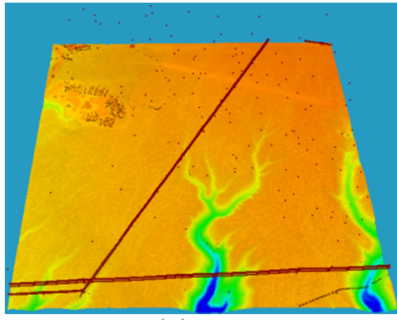

(a)

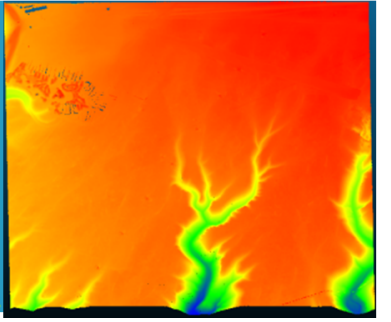

(b)
Figure 3. The 3D point cloud display of test data. (a) the raw point clouds; (b) the point cloud after preprocessing

\subsection{Experiment}

The purpose of this paper is to obtain the point cloud of the gully, so we select the point clouds that contain ravines from the experimental data. As shown in Fig. 4, 276461 points are selected and the average density of the point cloud is 6 points $/ \mathrm{m}^{2}$. The 3D diagram is shown in Fig. 4(a) and the imagery is shown in Fig. 4(b).

It can be seen from Fig. 4 that the gully feature is very irregular, the thalweg and the bottom line both belong to an irregular curve, and the height on the two sides of the ditch is not consistent. When applying the characteristic difference method, the appropriate distance radius must be used to choose the neighbourhood points of the experimental data. In order to obtain the proper distance radius, this paper experiments with radii of 1.5-4 m, considering that the point cloud average density is 6 points $/ \mathrm{m}^{2}$. The error threshold used in the experiment is set as $0.02 \mathrm{~m}$. The initial segmentation result is shown in Fig. 5 for the different radii.

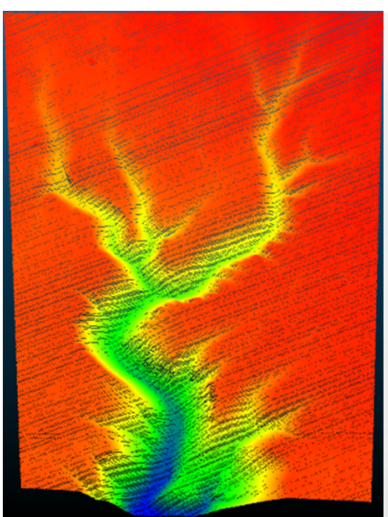

(a)

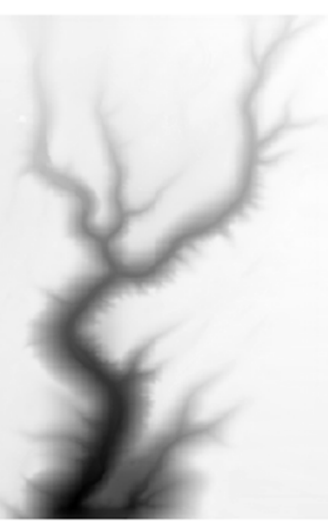

(b)
Figure 4. The show of test data. (a) the 3D point cloud; (b) the image

Figure 5 shows that there is an obvious difference in the initial segmentation result when using three different radii. On the whole, the general form of the gully can be identified, but the point clouds are discrete, especially on the edge of Fig. 5(b), and the point clouds that have gaps on both sides of the trench wall in Fig. 5(c) are less discrete. From Fig. 5(a), it can be seen that the segmentation result is superior to the two other results: the edge of the gully point cloud is in regular, and the density is homogeneous.

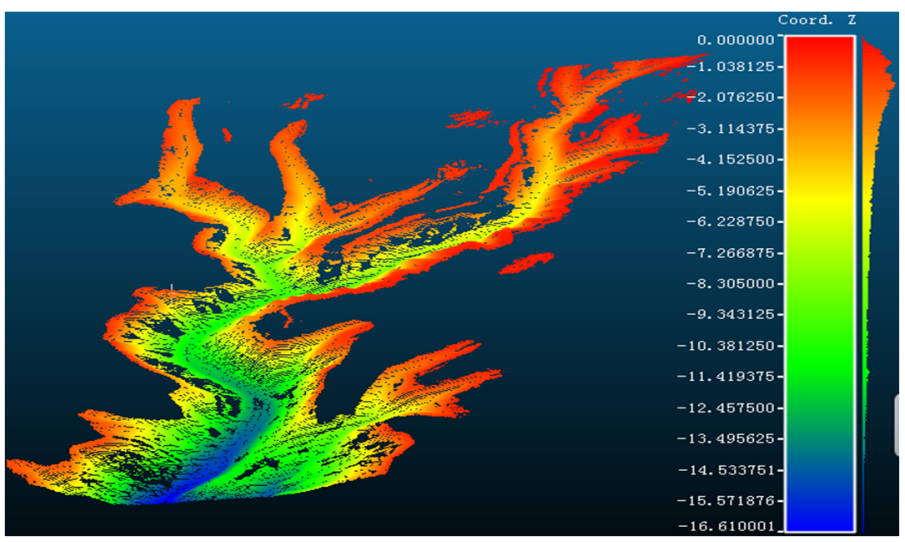

(a) $\mathrm{r} 1=4 \mathrm{~m}, \mathrm{r} 2=1.5 \mathrm{~m}$

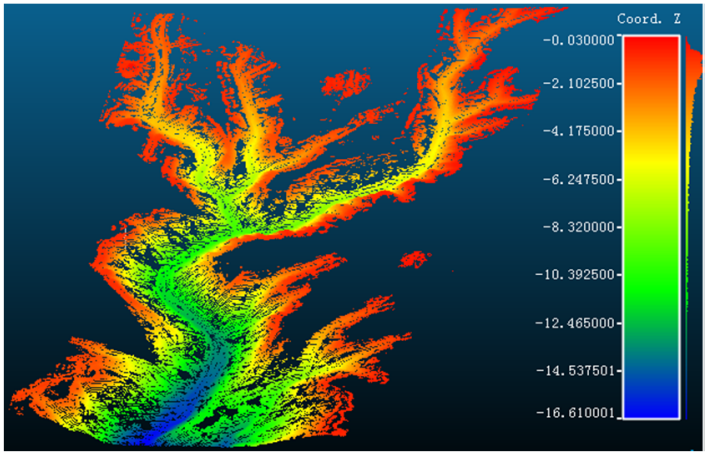

(b) $\mathrm{r} 1=3 \mathrm{~m}, \mathrm{r} 2=1.5 \mathrm{~m}$

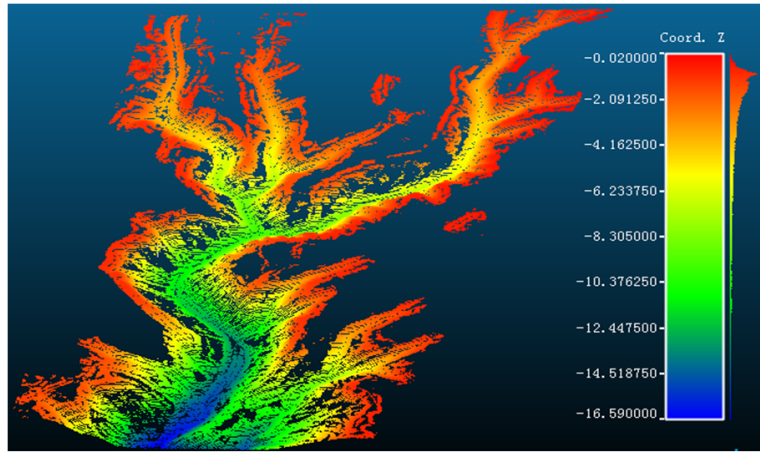

(c) $\mathrm{r} 1=5 \mathrm{~m}, \mathrm{r} 2=2 \mathrm{~m}$

Figure 5. The segmentation results of different radius 
In conclusion, the difference between two radii is not too big or too small. If the difference between two radii is too small, discretization of the point clouds is easy. If the difference between two radii is too big, it is easy to miss some points. According to the above test, from these data, 4 and $1.5 \mathrm{~m}$ are selected as the fixed distances in the process of initial segmentation. The experimental process is described below.

1) The efficiency of calculation is improved by the normalized processing of point clouds on the ground.

2) The neighbourhood points are chosen using radii of 4 and $1.5 \mathrm{~m}$ to calculate the normal vector and curvature values to get the characteristic difference value, and the error threshold is set as 0.02 to obtain the segmentation results, as shown in Fig. 5(a).

3) The clustering distance is set as $1 \mathrm{~m}$ to obtain the gully point cloud, as shown in Fig. 6.

The 103191 points that belong to the gully point clouds are obtained through the above experiment. Comparing Fig. 5(a) and Fig. 6, the points that do not belong to the gully are removed, and the rest of the point clouds not only keep the morphological features but also have an obvious edge.

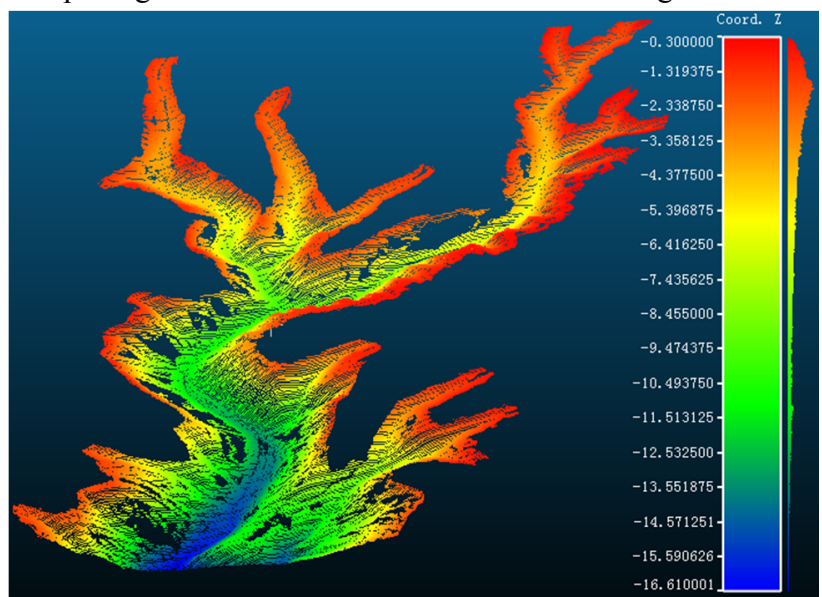

Figure 6 . The result of segmentation

\subsection{Parameter extraction of gully}

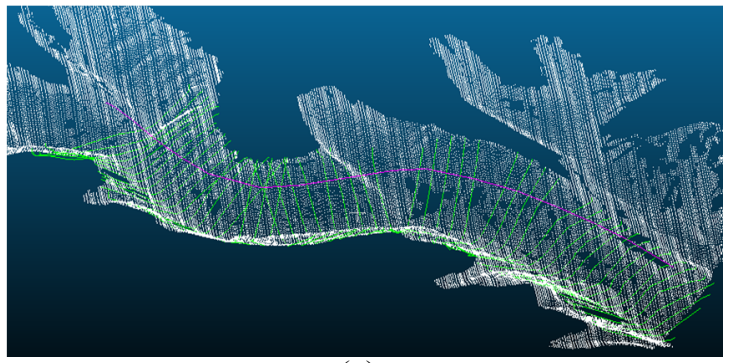

(a)

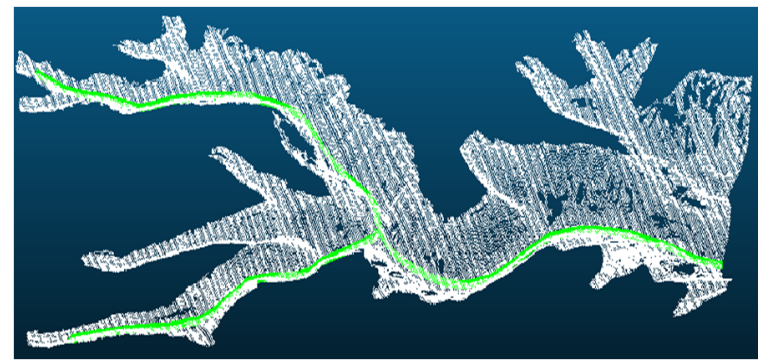

(b)

Figure 7. Extraction of gully parameters. (a)the cross-section profile of gully; (b) the bottom line of gully

In order to fully reflect the gully shape characteristic, the cross-section analysis proceeds in the gully point clouds that are obtained by segmentation as shown in Fig. 7. The spacing is set to $10 \mathrm{~m}$. Then the bottom line of the gully is measured, and the length is found to be $335 \mathrm{~m}$. The depth of the gully is shown in Fig. 8, the different selected places have different depths of $11.6 \mathrm{~m}, 6.2 \mathrm{~m}$, and $4.3 \mathrm{~m}$, respectively. The acquisition of the quantitative data on the gully parameters provides a new method for the quantitative evaluation and calculation of soil erosion in gullies.

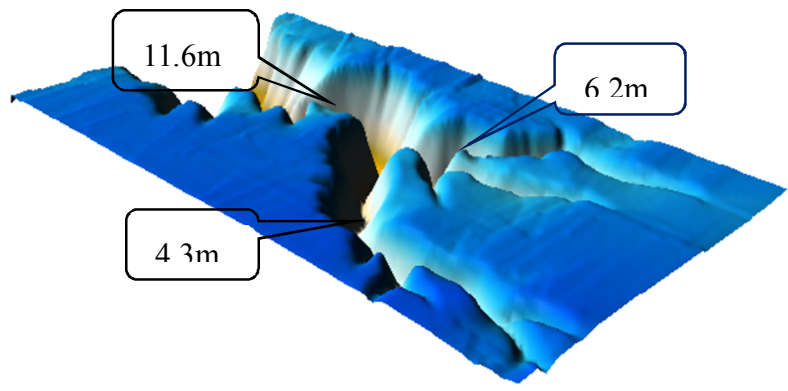

Figure 8 . The deep analysis of gully

\subsection{Analysis of results}

By the visualization carried out in this paper, it can be seen that extracted gully point clouds that are in line with the morphological characteristics of the gully are similar to the original point cloud form, as shown in Fig. 5. Other the extracted gully point clouds overlie the original point cloud that is in the gully position of the original point cloud as shown in Fig. 9(a). Moreover, 3D point clouds of the gully are combined with the DEM established by the original point cloud, and the distribution of the boundary of the gully is consistent with the elevation changes of the DEM, as shown in Fig. 9(b). Through the above experiments, information can be extracted from the gully point cloud relatively well and accurately.

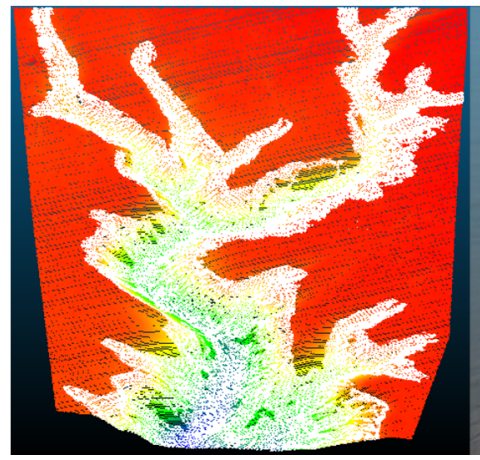

(a)

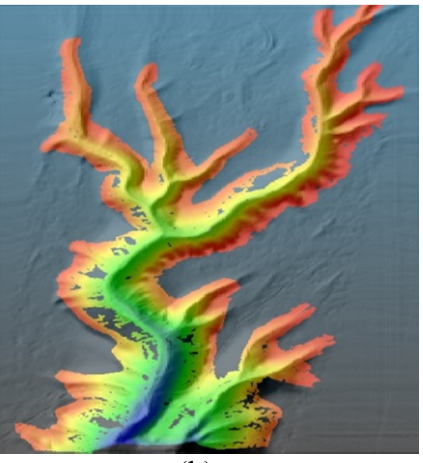

(b)
Figure 9. The verification of results for point cloud segmentation: (a) the overlay of raw point cloud and the point cloud of segmentation. (b) the overlay of DEM and the point cloud of segmentation

\section{CONCLUSIONS}

Because of the point cloud characteristic of the gully and the particularity of the terrain features, this paper puts forward a method of detecting airborne LIDAR gully point clouds based on the characteristic differences to compensate for a single scale using the characteristics of surface instability due to the multi-scale space from surface feature differences, which greatly improves the accuracy of extraction of the gully point cloud from the scattered point cloud. Use of the extracted gully 
point cloud to express the true form is reasonable and provides a basis for further evaluation of erosion. The radius and the setting of the rational threshold are key when the characteristic difference method is used to extract the gully point cloud. We need many experiments to determine a reasonable value because the numerical experiments utilized were an experience. The correctness of the evaluation to extract the gully point cloud can only be determined through comparison with images and manual extraction results and is currently difficult to analyze quantitatively. These problems need to be further studied in the future.

\section{ACKNOWLEDGMENTS}

This research has been financially supported by National Science Foundation under Grant No. 41174002 and the Open Research Found of Key Laboratory of Water Resources for the process and control of soil and water loss in loess plateau under Grant No. 2015003. The authors would like to thank the English editor for making improvements to the manuscript in its English form and are grateful to the anonymous referees.

\section{REFERENCES}

Betts, H. D., \& Derose, R. C, 1999. Digital elevation models as a tool for monitoring and measuring gully erosion. International Journal of Applied Earth Observation \& Geoinformation, 1(2), 91-101.

Baruch, A., \& Filin, S, 2011. Detection of gullies in roughly textured terrain using airborne laser scanning data. Isprs Journal of Photogrammetry \& Remote Sensing, 66(5), 564-578.

Douillard, B., Underwood, J., Melkumyan, N., \& Singh, S, 2010. Hybrid elevation maps: 3D surface models for segmentation. Ieee/rsj International Conference on Intelligent Robots and Systems (pp.1532-1538). IEEE Xplore.

Evans, M., \& Lindsay, J, 2010. High resolution quantification of gully erosion in upland peatlands at the landscape scale. Earth Surface Processes \& Landforms, 35(8), 876-886.

Filin, S., Avni, Y., Baruch, A., Morik, S., Arav, R., \& Marco, S, 2014. Characterization of land degradation along the receding dead sea coastal zone using airborne laser scanning. Geomorphology, 206(1), 403-420.

Pimentel, D., Harvey, C., Resosudarmo, P., Sinclair, K., Kurz, D., \& Mcnair, M., et al, 1995. Environmental and economic costs of soil erosion and conservation benefits. Science, 267(5201), 1117-23.

Lam, J., \& Greenspan, M, 2012. On the Repeatability of 3D Point Cloud Segmentation Based on Interest Points. Ninth Conference on Computer and Robot Vision (pp.9-16). IEEE Computer Society.

Mason, D. C., Scott, T. R., \& Wang, H. J, 2003. Extraction of tidal channel networks from airborne scanning laser altimetry. Isprs Journal of Photogrammetry \& Remote Sensing, 61(2), $67-83$.

Meng, X., Currit, N., \& Zhao, K, 2010. Ground filtering algorithms for airborne lidar data: a review of critical issues. Remote Sensing, 2(3), 833-860.

M., R. A., B., H. A., N., P. A., T., G. A., \& J., S. B. , 2014.
Object-based analysis of airborne laser scanning data for natural hazard purposes using open source components. International Archives of Photogrammetry, Remote Sensing and Spatial Information Sciences (Vol.19, pp.174-193).

Perroy, R. L., Bookhagen, B., Asner, G. P., \& Chadwick, O. A, 2010. Comparison of gully erosion estimates using airborne and ground-based lidar on santa cruz island, california. Geomorphology, 118(3), 288-300.

Tang, C. K., \& Medioni, G. , 2002. Curvature-augmented tensor voting for shape inference from noisy $3 \mathrm{~d}$ data. IEEE Transactions on Pattern Analysis \& Machine Intelligence, 24(6), 858-864.

Zhu, A. L., Wu, J., Xu, Z., Xu, Y., Lin, J., \& Hu, R., 2014. Coastline movement and change along the bohai sea from 1987 to 2012. Journal of Applied Remote Sensing, 8(1), 5230-5237.

Zhang, G. H., Xie, C. B., Xiao-Yu, P. I., \& Zuo, C. Q. , 2015. Effectiveness of soil conservation methods in preventing red soil erosion in southern china. Journal of Mountain Science, 12(5), 1281-1291.

Wu, Y., \& Cheng, H. , 2005. Monitoring of gully erosion on the loess plateau of china using a global positioning system. Catena, 63(2-3), 154-166. 